\title{
Diabetes mellitus prevents capsaicin from inducing hyperaemia in the rat sciatic nerve
}

\author{
D.W.Zochodne, L.T.Ho \\ Peripheral Nerve Research Laboratory, University of Calgary, Calgary, Alberta, Canada
}

\begin{abstract}
Summary. Loss of neurogenic inflammation in response to tissue injury may be an important complication of diabetes mellitus. We studied local neurogenic inflammation in the peripheral nerve trunk of Sprague-Dawley rats 4 months following the induction of diabetes by streptozotocin injection. To assess neurogenic inflammation, the epineurial plexus of the sciatic nerve was exposed to topical capsaicin, an agent that releases vasoactive neuropeptides from perivascular afferent terminals. Under normal circumstances, local vasodilatation results in endoneurial hyperaemia or a 'flare'. We evaluated the influence of capsaicin in diabetic sciatic nerve by making serial measurements of endoneurial blood flow using microelectrodes sensitive to hydrogen clearance. After 4 months of hyperglycaemia (glucose $>16.0 \mathrm{mmol} / \mathrm{l}$ ), diabetic animals had slowing of unmyelinated and myeli-
\end{abstract}

nated sural sensory conduction velocity compared to citrate buffer injected controls. Baseline sciatic endoneurial blood flow was unaltered by diabetes, and was comparable to controls. There was an expected hyperaemic response of endoneurial blood flow to capsaicin in control rat sciatic endoneurium but no consistent 'flare' response in diabetic rats. Our findings indicate that there is loss of capsaicin-related neurogenic inflammation in the vasa nervorum of experimental diabetes. It is possible that a similar deficit following nerve injury could impair the milieu for axonal regeneration in diabetes.

Key words: Diabetic neuropathy, vasa nervorum, neurogenic inflammation.
In skin and other tissues, there is evidence that diabetes impairs microcirculatory responses to injury, a feature that may contribute toward poor healing [1]. In particular, an important response to injury is the development of neurogenic inflammation, in which afferent nerve fibres release local peptides that increase local blood flow, allow plasma extravasation, and recruit immunomodulatory cells [2]. Substance $\mathrm{P}(\mathrm{SP})$, calcitonin gene-related peptide (CGRP), neurokinin A and neurokinin $B$ are among the nerve terminal peptides that participate in these local responses [3]. SP and CGRP, in concert with histamine that they help release from local mast cells, are local vasodilators of microvessels, probably acting through nitric oxide [4-7]. In diabetes, the local neurogenic flare response, perhaps mediated by an 'axon reflex' may be impaired $[8$, 9].

Diabetic peripheral nerve recovers slowly following focal injury but the factors that contribute toward impaired regeneration are not understood. Aldose reductase inhibitors correct metabolic abnormalities and improve nerve conduction but fail to improve regeneration in diabetic nerves [10]. Hypoxia and reduced local blood flow have been observed in uninjured nerves of animals with experimental diabetic neuropathy and could contribute poor recovery $[11,12]$. Not all reports, however, have identified these reductions in blood flow [13-15].

A model of nerve trunk 'neurogenic inflammation' is that associated with the acute action of capsaicin, an agent that depolarizes peptidergic afferent fibres, including perivascular terminals [16]. These terminals then release several peptides including SP, and CGRP that mediate a local flare response, in concert with histamine from mast cells [3]. Applied to the epineurial plexus, capsaicin induces an intense and prolonged hyperaemic response, a 'peripheral nerve trunk flare' [16].

In this work, we evaluated capsaicin-induced neurogenic inflammation in vasa nervorum of streptozotocin (STZ)-induced diabetic rats. The response to capsaicin was evaluated by making local and serial measurements of endoneurial blood flow (EBF) using hydrogen-sensitive microelectrodes. In addition we made electrophysiological measurements of sural myelinated and unmyelinated conduction to assess the degree of associated neuropathy. 
Table 1. Final body weights and blood glucose values 4 months following streptozotocin (diabetic rats) or citrate buffer (control)

\begin{tabular}{lll}
\hline & Diabetic rats & Control rats \\
\hline$n$ & 10 & 8 \\
Weight $(\mathrm{g})$ & $266 \pm 14^{\mathrm{a}}$ & $611 \pm 21$ \\
Glucose $(\mathrm{mmol} / \mathrm{)})$ & $23.8 \pm 1.6^{\mathrm{a}}$ & $6.9 \pm 0.3$ \\
\hline
\end{tabular}

${ }^{\mathrm{a}} p<0.0001$ vs control rats, Values are means $\pm \mathrm{SEM}$

Table 2. Sural nerve conduction studies

\begin{tabular}{lll}
\hline & Diabetic rats & Control rats \\
\hline Myelinated conduction & & \\
$n$ & 8 & 6 \\
$\mathrm{CV}(\mathrm{m} / \mathrm{s})$ & $53.4 \pm 1.9^{\mathrm{a}}$ & $64.9 \pm 3.7$ \\
Amplitude $(\mathrm{mV})$ & $2.45 \pm 0.49$ & $2.93 \pm 0.68$ \\
Unmyelinated conduction & & \\
$n$ & 6 & 5 \\
$\mathrm{CV}(\mathrm{m} / \mathrm{s})$ & $1.04 \pm 0.08^{\mathrm{b}}$ & $1.50 \pm 0.09$ \\
Amplitude $(\mu \mathrm{V})$ & $50.5 \pm 15.0$ & $59.3 \pm 17.7$ \\
\hline
\end{tabular}

${ }^{\mathrm{a}} p=0.006,{ }^{\mathrm{b}} p=0.002$ vs control rats. Results are means $\pm \mathrm{SEM}$. $\mathrm{CV}$, Conduction velocity

Table 3. Endoneurial blood flow, microvascular resistance and mean arterial pressure

\begin{tabular}{|c|c|c|}
\hline & Diabetic rats & Control rats \\
\hline$n$ & 10 & 8 \\
\hline \multicolumn{3}{|c|}{$\begin{array}{l}\text { Endoneurial blood flow } \\
\left(\mathrm{ml} \cdot 100 \mathrm{~g}^{-1} \cdot \mathrm{min}^{-1}\right)\end{array}$} \\
\hline Pre-capsaicin & $19.6 \pm 2.1$ & $16.9 \pm 2.1^{a}$ \\
\hline Post-capsaicin & $22.5 \pm 2.5$ & $23.6 \pm 2.5$ \\
\hline \multicolumn{3}{|c|}{$\begin{array}{l}\text { Endoneurial microvascular resistance } \\
\quad\left(\mathrm{mm} \mathrm{Hg} \cdot 100 \mathrm{~g} \cdot \mathrm{min} \cdot \mathrm{mt}^{-1}\right)\end{array}$} \\
\hline Pre-capsaicin & $6.67 \pm 0.82$ & $7.71 \pm 1.12^{b}$ \\
\hline Post-capsaicin & $5.83 \pm 0.53$ & $5.87 \pm 0.59$ \\
\hline \multicolumn{3}{|c|}{$\begin{array}{l}\text { Mean arterial pressure } \\
\quad(\mathrm{mm} H \mathrm{Hg})\end{array}$} \\
\hline Pre-capsaicin & $117 \pm 5$ & $116 \pm 5^{c}$ \\
\hline Post-capsaicin & $120 \pm 3$ & $129 \pm 1$ \\
\hline
\end{tabular}

${ }^{\mathrm{a}} p=0.003,{ }^{\mathrm{b}} p=0.037$ pre-vs post-capsaicin, ${ }^{\mathrm{c}} p=0.03$ pre-vs postcapsaicin (two-tailed test). Results are means \pm SEM

\section{Materials and methods}

Male Sprague-Dawley rats of initial weight $250-350 \mathrm{~g}$ were given i.p. injections of $S T Z(65 \mathrm{mg} / \mathrm{kg})$ in citrate buffer at $\mathrm{pH} 4.5$ and accepted as diabetic if blood glucose was greater than or equal to $16.0 \mathrm{mmol} / \mathrm{l}$ (measured 5-7 days after STZ). Controls were injected with citrate buffer alone. Glucose measurements were made from the ventral caudal vein using a glucometer (Accu-Chek IIm; Boehringer Mannheim Canada, Dorval, Quebec, Canada) and the oxygen rate method (Beckman Glucose Analyser 2; Beckman Instruments Inc., Palo Alto, Calif., USA). Rats were housed in grouped wire cages with free access to rat chow and water. After 16 weeks of diabetes, measurements of in vitro sural sensory conduction velocity and compound nerve action potential amplitudes were made in myelinated and unmyelinated fibres at $37^{\circ} \mathrm{C}$. The electrophysiological techniques have been described in our previous work [14].

Electrophysiological studies and blood flow studies were conducted in rats anaesthetized with sodium pentobarbital $(65 \mathrm{mg} / \mathrm{kg}$ i.p.). The rats were then prepared for local measurements of endo- neurial blood flow in the contralateral sciatic nerve using microelectrodes linearly sensitive to hydrogen clearance. Our experimental preparation has previously been described [16]. In brief, anaesthetized rats (as above) underwent tracheostomy and placement of a left carotid intra-arterial catheter, then were paralysed using tubocurarine (1.5 $\mathrm{mg} / \mathrm{kg}$ intra-arterial) and ventilated. Arterial blood gases were drawn from the catheter every $30-60 \mathrm{~min}$ to ensure that values were within an acceptable physiological range (i.e. $\mathrm{pO}_{2}>90$ torr; $\mathrm{pCO}_{2}$ 35-45 torr; $\mathrm{pH}$ 7.35-7.45). Mean arterial blood pressure (MAP) was continuously recorded from the carotid catheter. Supplemental doses of pentobarbital $(20 \mathrm{mg} / \mathrm{kg})$ were given approximately every $2 \mathrm{~h}$ to maintain a stable level of anaesthesia (as judged by the level and stability of MAP). Local EBF was measured in the mid portion of the sciatic nerve just above its trifurcation. The left sciatic nerve was exposed, covered in mineral oil maintained at $37^{\circ} \mathrm{C}$, and a linearly sensitive hydrogen microelectrode inserted through an epineurial window into the endoneurium. Hydrogen was added to the ventilatory gas mixture until endoneurial saturation, then turned off to permit recording of the hydrogen clearance curve. Curves were fitted to mono- or biexponential models to provide EBF measurements using a least squares regression program as previously described [16]. Endoneurial microvascular resistance (EMR) was calculated as MAP/EBF.

In each animal, three clearance curves were recorded. The first curve was routinely discarded, and the second served as a baseline control measurement. As in previous work, the third curve was preceded by bathing the epineurium with $2 \%$ capsaicin in $10 \%$ ethanol and $10 \%$ Tween 80 . The carrier does not significantly alter hydrogen clearance [16]. Capsaicin was applied to the epineurium $5 \mathrm{~min}$ before the onset of the clearance curve and was left in situ over the epineurium until hydrogen clearance was completed.

\section{Statistical analysis}

Data were analysed by determining means and standard errors for electrophysiological results, EBF, MAP and EMR and changes in EBF and EMR following capsaicin. Diabetic and non-diabetic electrophysiological results were compared using an unpaired one-tailed Student's $t$-test. Choice of the one-tailed test was justified by the expected direction of change in these parameters with diabetes [13]. Paired one-tailed Student's $t$-tests were used to compare EBF and EMR measurements before and after capsaicin. In previous work using non-diabetic animals, capsaicin was associated with a rise in EBF and a fall in EMR [16] justifying the choice of a one-tailed test in the present work.

\section{Results}

Diabetic rats gained less weight than controls (Table 1), and developed cataracts. Conduction velocities in sural myelinated and unmyelinated fibres were significantly lower in diabetic rats than controls after 16 weeks of diabetes. There was a non-significant trend toward lower myelinated fibre compound nerve action potential amplitudes in diabetics but unmyelinated fibre ' $\mathrm{C}$ ' potentials were comparable in amplitude between the groups (Table 2). Baseline EBF, EMR and MAP were statistically similar between diabetic and non-diabetic controls. Following the application of epineurial capsaicin, there was a significant rise in EBF (increased by $28 \%$ ) and a fall in EMR (fell by $24 \%$ ) over baseline measurements in non-diabetic animals, indicating vasodilation (Table 3 ). These changes were similar to results previously obtained in non-diabetic rats (EBF increased by $37 \%$ and EMR fell by $23 \%$ [16]). A mild rise in MAP accompanied these 
changes. Although mean EBF rose slightly and mean EMR fell slightly in diabetic animals after capsaicin, changes in individual animals were inconsistent and the difference between pre- and post-capsaicin EBF and EMR were not statistically significant (Table 3 ).

\section{Discussion}

The findings of this study were: 1 . diabetic rats developed expected slowing of sural myelinated sensory fibre conduction but also had slowing of unmyelinated fibre conduction; 2. baseline endoneurial blood flow was not altered by diabetes (there was a non-significant trend toward higher flow in the diabetic animals); 3 . there was no consistent hyperaemic response to epineurial capsaicin in diabetic rats.

Although mean EBF in diabetic rats increased slightly after capsaicin, a consistent and statistically significant rise did not occur. These findings are not unexpected. Several mechanisms could interrupt capsaicin-induced peptide hyperaemia in diabetes. Neuropathy could reduce the numbers of perivascular nerve terminals or their contents of CGRP (calcitonin gene-related peptide), and SP (substance $P$ ) that are released by capsaicin. Our findings indicate that there is involvement of unmyelinated fibres in diabetes: perivascular fibres in the epineurial plexus could indeed be abnormal. Reduction of the content of these peptides in nerve terminals or dystrophic changes in their axons from other tissues of diabetic animals have been noted [17, 18]. Milner et al. [19], however, found increased, rather than decreased SP and CGRP levels in the sheaths of sciatic nerves from rats having diabetes for 8 weeks.

It is possible that perivascular nerve terminals are insensitive to the depolarizing action of capsaicin or that diabetic microvessels become mechanically rigid and lose their capacity to dilate under physiological conditions. In human diabetic sural nerve, microvessels have basement membrane thickening and there is evidence that endoneurial collagen, possibly through glycation, forms more rigid endoneurial structures [20-22]. Other features of diabetic microangiopathy could include non-specific alterations in the smooth muscle contractile apparatus, the availability of calcium, changes in smooth muscle receptor properties or other abnormalities [23]. Indeed, several studies have identified abnormalities in the vasoreactivity and appearance of large and small blood vessels in diabetic tissues indicating the presence of a 'functional microangiopathy' [24-30]. Gamse et al. [31], noted that STZ diabetes in rats abolished both plasma extravasation in rat abdominal skin induced by the direct application of SP and the potentiation of the SP action by CGRP. These findings might suggest that there is an alteration of peptide receptors on mast cells, a change in mast cell numbers, or direct changes of blood vessels in diabetes.

Dysfunction of endothelial mediated vasodilation and contraction could also account for a 'functional microangiopathy'. A common defect at the endothelial level was suggested by Mayhan [27]. SP, CGRP and histamine mediate vasodilation through intact endothelium by releasing nitric oxide [4-6]. Recent evidence has indicated that nitric oxide is quenched by advanced glycation products and inactivated [32]. Finally, endothelial cell dysfunction in diabetes might also impair the generation of nitric oxide [5].

Lowered baseline EBF using hydrogen clearance has been reported in a model of STZ-induced diabetic neuropathy similar to ours $[11,12]$. Although this finding has apparently been confirmed by other groups [33,34], technical considerations may not render the confirmation valid. Normal 'baseline' EBF has been a consistent feature of our studies in large numbers of rats rendered diabetic by STZ $[13,14]$ or in BB Wistar rats with genetic diabetes (Zochodne and $\mathrm{Ho}$, unpublished data). Normal nerve EBF has also been reported by Pugliese et al. [15] and morphological appearances of microvessels in experimental diabetes (in contrast to functional changes discussed above) have been unimpressive [35]. It is possible that these discrepancies among investigators may result from other factors: levels of hyperglycaemia in the rats, differences in the genetic strains of the rats used, or variations in the physiological status of the rats. It is also possible that our normal baseline hydrogen clearance EBF measurements could have missed subtle microangiopathy. Plasma skimming of erythrocytes at microvascular bifurcations could result in the clearance of hydrogen by plasma alone [36]. Despite these problems hydrogen clearance provides selective quantitative blood flow information about the endoneurial nutritive circulation and permits serial comparison measurements as in this work. Nonetheless, it would also be important to evaluate the capsaicin flare in genetic diabetes of rats, where the STZ toxin has not been given (although it is unlikely to have influenced vascular responsiveness 4 months after its administration and does not alter nerve conduction without diabetes [14]).

Thus, although there is powerful evidence that microangiopathy does occur in diabetes, an aetiological relationship between changes in nerve perfusion and conduction abnormalities in experimental diabetes remains uncertain. Microangiopathy, however, could play a critical role in the response of nerve to injury.

Our finding that there was loss of the capsaicin 'flare' in diabetic peripheral nerve is consistent with findings in other diabetic tissues that neurogenic inflammation is impaired $[8,9,31]$. Following tissue injury, a Lewis flare is mediated through an 'axon reflex' or 'axon response' accomplished by the perivascular afferent terminal release of SP and CGRP and histamine release by mast cells. In non-diabetic animals, there is evidence that local blood flow is maintained and enhanced following focal nerve injury partly through the action of CGRP $[37,38]$. Thus, a 'neurogenic flare' may also be an important component of the peripheral nerve trunk response to injury. Local peptidergic actions may thus have important compensatory functions in injury, such as increasing local tissue content of oxygen, recruiting of macrophages and allowing entry of trophic agents through the normally tight blood-nerve barrier. Loss of the neurogenic injury flare in diabetes could slow regenerative events attempted by endoneurial axons. 
Acknowledgements. The work was supported by funding from the Medical Research Council of Canada and the Muscular Dystrophy Association of Canada. DWZ received support as a Career Scientist of the Ontario Ministry of Health and is currently a Clinical Investigator of the Alberta Heritage Foundation for Medical Research. Ms $\mathrm{E}$. Wong and $\mathrm{Ms} \mathrm{H}$. Price provided secretarial assistance.

\section{References}

1. Walmsley D, Wales JK, Wiles PG (1989) Reduced hyperaemia following skin trauma: evidence for an impaired microvascular response to injury in the diabetic foot. Diabetologia 32: 736-739

2. Lembeck F (1983) Sir Thomas Lewis's nocifensor system, histamine and substance-P-containing primary afferent nerves. Trends Neurosci 6: 106-108

3. Holzer P (1988) Local effector functions of capsaicin-sensitive sensory nerve endings: involvement of tachykinins, calcitonin gene-related peptide and other neuropeptides. Neuroscience 24: $739-768$

4. Fiscus RR, Zhou H-X, Wang X et al. (1991) Calcitonin gene-related peptide (CGRP)-induced cyclic AMP, cyclic GMP and vasorelaxant responses in rat thoracic aorta are antagonized by blockers of endothelium-derived relaxant factor (EDRF). Neuropeptides 20: 133-143

5. Luscher TF, Vanhoutte PM (1990) The endothelium: modulator of cardiovascular function. CRCPress, Boca Raton, p. 4

6. Piotrowski W, Foreman JC (1986) Some effects of calcitonin gene-related peptide in human skin and on histamine release. $\mathrm{Br}$ J Dermat 114: $37-46$

7. Kiernan JA (1975) A pharmacological and histological investigation of the involvement of mast cells in cutaneous axon reflex vasodilatation. Q J Exp Physiol 60: 123-130

8. Parkhouse N, Le Quesne PM (1988) Impaired neurogenic vascular response in patients with diabetes and neuropathic foot lesions. N Engl J Med 318: 1306-1309

9. Hutchison KJ, Johnson BW, Williams HTG, Brown GD (1974) The histamine flare response in diabetes mellitus. Surgery Gynecol Obstet 139: 566-568

10. Ekstrom PAR, Tomlinson DR (1989) Impaired nerve regeneration in streptozotocin-diabetic rats. J Neurol Sci 93: 231-237

11. Tuck RR, Schmelzer JD, Low PA (1984) Endoneurial blood flow and oxygen tension in the sciatic nerves of rats with experimental diabetic neuropathy. Brain 107: 935-950

12. Cameron NE, Cotter MA, Low PA (1991) Nerve blood flow in early experimental diabetes in rats: relation to conduction deficits. Am J Physiol 261: E1-E8

13. Zochodne DW, Ho LT (1992) Normal blood flow but lower oxygen tension in diabetes of young rats: microenvironment and the influence of sympathectomy. Can J Physiol Pharmacol 70: $651-659$

14. Zochodne DW, HoLT (1992) The influence of indomethacin and guanethidine on experimental streptozotocin diabetic neuropathy. Can J Neuro Sci 19:433-441

15. Pugliese G, Tilton RG, Speedy A et al. (1989) Effects of very mild versus overt diabetes on vascular haemodynamics and barrier function in rats. Diabetologia 32: 845-857

16. Zochodne DW, Ho LT (1991) Influence of perivascular peptides on endoneurial blood flow and microvascular resistance in the sciatic nerve of the rat. J Physiol (London) 444:615-630

17. Willars GB, Calcutt NA, Compton AM, Tomlinson DR, Keen P (1989) Substance $P$ levels in peripheral nerve, skin, atrial myocardium and gastrointestinal tract of rats with long-term diabetes mellitus. J Neurol Sci 91: 153-164

18. Schmidt RE, Plurad DA, Plurad SB, Cogswell BE, Diani AR, Roth KA (1989) Ultrastructural and immunohistochemical characterization of autonomic neuropathy in genetically diabetic Chinese hamsters. Lab Invest 61:77-92

19. Milner P, Appenzeller O, Qualls C, Burnstock G (1992) Differential vulnerability of neuropeptides in nerves of the vasa nervorum to streptozotocin-induced diabetes. Brain Res 574: $56-62$

20. Malik RA, Newrick PG, Sharma AK et al. (1989) Microangiopathy in human diabetic neuropathy: relationship between capillary abnormalities and the severity of neuropathy. Diabetologia 32: $92-102$

21. Brownlee M, Cerami A, Vlassara H (1988) Advanced glycosylation end products in tissue and the biochemical basis of diabetic complications. NEngl J Med 318: 1315-1321

22. King RHM, Llewelyn JG, Thomas PK, Gilbey SG, Watkins PJ (1989) Diabetic neuropathy: abnormalities of Schwann cell and perineurial basal laminae. Implications for diabetic vasculopathy. Neuropathol Appl Neurobiol 15: 339-355

23. Lee T-S, Saltsman KA, Ohashi H, King GL (1989) Activation of protein kinase $\mathrm{C}$ by elevation of glucose concentration: proposal for a mechanism in the development of diabetic vascular complications. Proc Natl Acad Sci USA 86: 5141-5145

24. Sullivan S, Sparks HV (1979) Diminished contractile response of aortas from diabetic rabbits. Am J Physiol 236: H301-H306

25. Pfaffman MA, Ball CR, Darby A, Hilman R (1982) Insulin reversal of diabetes-induced inhibition of vascular contractility in the rat. Am J Physiol 242: H490-H495

26. Turlapaty PDMV, Lum G, Altura BM (1980) Vascular responsiveness and serum biochemical parameters in alloxan diabetes mellitus. Am J Physiol 239: E412-E421

27. Mayhan WG (1989) Impairment of endothelium-dependent dilatation of cerebral arterioles during diabetes mellitus. Am J Physiol 256: H621-H625

28. Williamson JR, Ostrow E, Eades D et al. (1990) Glucose-induced microvascular functional changes in non-diabetic rats are stereospecific and are prevented by an aldose reductase inhibitor. J Clin Invest 85: 1167-1172

29. Bohlen $\mathrm{HG}$, Niggl BA (1980) Early arteriolar disturbances following streptozotocin-induced diabetes mellitus in adult mice. Microvasc Res 20: 19-29

30. Bohlen HG, Hankins KD (1982) Early arteriolar and capillary changes in streptozotocin-induced diabetic rats and intraperitoneal hyperglycaemic rats. Diabetologia 22:344-348

31. Gamse R, Posch M, Saria A, Jancso G (1987) Several mediators appear to interact in neurogenic inflammation. Acta Physiologica Hungarica 69: 343-354

32. Bucala R, Tracey KJ, Cerami A (1991) Advanced glycosylation products quench nitric oxide and mediate defective endothelium-dependent vasodilatation in experimental diabetes. J Clin Invest 87: 432-438

33. Yasuda H, Sonobe M, Yamashita M et al. (1989) Effect of prostaglandin E1 analogue TFC612 on diabetic neuropathy in streptozocin-induced diabetic rats. Comparison with aldose reductase inhibitor ONO 2235. Diabetes 38: 832-838

34. Monafo WW, Eliasson SG, Shimazaki S, Sugimoto H (1988) Regional blood flow in resting and stimulated sciatic nerve of diabetic rats. Exp Neurol 99: 607-614

35. Sharma AK, Thomas PK (1974) Peripheral nerve structure and function in experimental diabetes. J Neurol Sci 23: 1-15

36. Pries AR, Ley K, Claassen M, Gaehtgens P (1989) Red cell distribution at microvascular bifurcations. Microvasc Res 38: 81101

37. Zochodne DW, Ho LT (1990) Endoneurial microenvironment and acute nerve crush injury in the rat sciatic nerve. Brain Res 535: $43-48$

38. Zochodne DW, Ho LT (1992) Hyperemia of injured peripheral nerve: sensitivity to CGRP antagonism. Brain Res 598: 59 66

Received: 30 November 1992

and in revised form: 15 February 1993

Dr. D. W.Zochodne

Department of Cinical Neurosciences

University of Calgary

Room 182 A, Heritage Medical Research Building

3330 Hospital Drive N.W.

Calgary, Alberta, Canada T2N 4N1 